\title{
Somatosensory evoked potentials in patients with high-grade aneurysmal subarachnoid hemorrhage
}

\author{
Klaus Christian Mende, MD, ${ }^{1}$ Mathias Gelderblom, MD, ${ }^{2}$ Cindy Schwarz, MTA, ${ }^{1}$ \\ Patrick Czorlich, MD, ${ }^{1}$ Nils Ole Schmidt, MD, ${ }^{1}$ Eik Vettorazzi, MSc, ${ }^{3}$ Jan Regelsberger, MD, \\ Manfred Westphal, MD, ${ }^{1}$ and Tammam Abboud, MD ${ }^{4}$
}

Departments of ${ }^{1}$ Neurosurgery, ${ }^{2}$ Neurology, and ${ }^{3}$ Medical Biometry and Epidemiology, University Medical Center Hamburg-Eppendorf, Hamburg; and ${ }^{4}$ Department of Neurosurgery, University Medical Center Göttingen, Germany

\begin{abstract}
OBJECTIVE The aim of this prospective study was to investigate the value of somatosensory evoked potentials (SEPs) in predicting outcome in patients with high-grade aneurysmal subarachnoid hemorrhage (SAH).

METHODS Between January 2013 and January 2015, 48 patients with high-grade SAH (Hunt and Hess Grade III, IV, or V) who were admitted within 3 days after hemorrhage were enrolled in the study. Right and left median and tibial nerve SEPs were recorded on Day 3 after hemorrhage and recorded again 2 weeks later. Glasgow Outcome Scale (GOS) scores were determined 6 months after hemorrhage and dichotomized as poor (Scores 1-3) or good (Scores 4-5). Results of SEP measurements were dichotomized (present or missing cortical responses or normal or prolonged latencies) for each nerve and side. These variables were summed and tested using logistic regression and a receiver operating characteristic curve to assess the value of SEPs in predicting long-term outcome.

RESULTS At the 6-month follow-up visit, 29 (60.4\%) patients had a good outcome, and 19 (39.6\%) had a poor outcome. The first SEP measurement did not correlate with clinical outcome (area under the curve [AUC] $0.69, p=0.52$ ). At the second measurement of median nerve SEPs, all patients with a good outcome had cortical responses present bilaterally, and none of them had bilateral prolonged latencies ( $p=0.014$ and 0.003 , respectively). In tibial nerve SEPs, $7.7 \%$ of the patients with a good GOS score had one or more missing cortical responses, and bilateral prolonged latencies were found in $23 \%$ ( $p=0.001$ and 0.034 , respectively). The second measurement correlated with the outcome regarding each of the median and tibial nerve SEPs and the combination of both (AUC 0.75 [p = 0.010], 0.793 [p $=0.003$ ], and 0.81 [p = 0.001], respectively).
\end{abstract}

CONCLUSIONS Early SEP measurement after SAH did not correlate with clinical outcome, but measurement of median and tibial nerve SEPs 2 weeks after a hemorrhage did predict long-term outcome in patients with high-grade SAH. https://thejns.org/doi/abs/10.3171/2017.7.FOCUS17427

KEY WORDS intracranial aneurysm; subarachnoid hemorrhage; somatosensory evoked potentials

$\mathrm{S}$ UBARACHNOID hemorrhage (SAH) after aneurysm rupture is a devastating disease. It has an incidence of 9 cases per 100,000 patient-years ${ }^{17}$ and occurs most commonly in patients between 40 and 60 years of age. ${ }^{4}$ Improvements in the therapy for aneurysmal SAH over the past 3 decades have led to a reduction in the case fatality rate to approximately $35 \% .{ }^{17}$ Estimation of the severity of initial brain injury and anticipation of the clinical outcome currently are based on neurological examination and neuroimaging studies. However, the clinical evaluation of patients with high-grade SAH is often difficult because of the sedatives and analgesic medications that frequently are given to these patients. Moreover, second- ary complications, such as delayed cerebral ischemia after vasospasm, which might develop during the course of the disease, frequently lead to a longer hospital stay and affect the clinical outcome.

Measurement of somatosensory evoked potentials (SEPs) is an established neurophysiological technique for estimating neurological function. This measurement usually is available at bedside and can be repeated easily without significant risk to the patient. Its role in predicting unfavorable outcome in comatose patients in general ${ }^{8,13}$ and after cardiac arrest ${ }^{16}$ or stroke ${ }^{2,7,24}$ in particular has been well studied. Several studies found that early median nerve SEPs after initial admission predicts brain death, ${ }^{8,19}$ but none of them

ABBREVIATIONS AUC = area under the curve; GCS = Glasgow Coma Scale; GOS = Glasgow Outcome Scale; HH = Hunt and Hess; ROC = receiver operating characteristic; $\mathrm{SAH}=$ subarachnoid hemorrhage; $\mathrm{SEP}=$ somatosensory evoked potential.

SUBMITTED June 29, 2017. ACCEPTED July 24, 2017.

INCLUDE WHEN CITING DOI: 10.3171/2017.7.FOCUS17427. 
used tibial nerve SEPs for this purpose. Furthermore, the value of SEPs in patients with SAH as a distinct group has not been addressed yet. Therefore, we designed this prospective study to analyze SEPs in patients with high-grade $\mathrm{SAH}$ at 2 different points to investigate their correlation with long-term clinical outcome and compare them with the established clinical parameters. Another goal of this study was to determine whether tibial nerve SEPs can add useful information regarding clinical outcome.

\section{Methods \\ Patient Population}

Every patient with SAH who was admitted to the intensive care unit of the University Medical Center HamburgEppendorf between January 2013 and January 2015 was screened for this study. Included were patients older than 18 years with newly diagnosed SAH, Grade III or higher on the Hunt and Hess (HH) scale, ${ }^{10}$ if the hemorrhage took place within 3 days before admission. Patients with recurrent hemorrhage, concomitant trauma, or preexisting myelopathy or polyneuropathy were excluded from the study.

Each patient was admitted to the intensive care unit and underwent at least $1 \mathrm{CT}$ study at the time of admission. Ventriculostomy was performed when clinical signs of hydrocephalus were present or hydrocephalus was visible on the CT scan. Afterward, aneurysm repair was performed by either endovascular or microsurgical means based on the decision of an interdisciplinary board that included a team of senior neurosurgeons and interventional neuroradiologists. After aneurysm repair, the goal of treatment was to reduce sedation and attempt to extubate the patient when possible to enable clinical evaluation. In addition to normovolemia and maintenance of a mean arterial blood pressure higher than $90 \mathrm{~mm} \mathrm{Hg}$, each patient was treated with intravenous nimodipine for the 1st week after the hemorrhage and then oral nimodipine for 2 weeks. We screened for vasospasm by performing transcranial Doppler ultrasonography daily; if mean flow velocity increased by $50 \mathrm{~cm} / \mathrm{sec}$ or $100 \%$ within 24 hours or if a threshold of $200 \mathrm{~cm} / \mathrm{sec}$ was exceeded, cerebral angiography was performed, and intraarterial nimodipine was administered when vasospasm was detected. After the acute treatment period, patients were referred to a specialized neurorehabilitation unit either directly or after a short stay in the neurological or neurosurgical ward.

\section{Clinical Parameters}

Basic demographic data, initial Glasgow Coma Scale (GCS) score, ${ }^{11,21} \mathrm{HH}$ grade, and blood distribution on initial CT scans according to the Fisher scale were recorded. To determine patient outcome, the Glasgow Outcome Scale (GOS) score was determined at discharge and at the 6-month follow-up visit. For statistical analysis, dichotomization was used (good outcome [GOS score of 4 or 5] or poor outcome [GOS score of 1,2, or 3]).

\section{Somatosensory Evoked Potentials}

Bedside recording of SEPs from the right and left median nerves and from the right and left tibial nerves was performed within 3 days after hemorrhage and repeated
14 days later. Measurements were performed by a medical technologist with dedicated training in neurophysiological diagnosis using the Nihon Kohden system (Neuropack M1 and polaris.one); a dual-channel setup for the median nerve and single-channel setup for the tibial nerve were used. Stimulus rates of 2 and $3 \mathrm{~Hz}$ were used for median and tibial nerve SEPs, respectively. The stimulus intensity was set at $3 \mathrm{~mA}$ over the threshold that elicited a visible twitch of the thumb or toe muscles with a pulse duration of $0.2 \mathrm{msec}$. An average of 100-200 responses were recorded with at least 2 repetitions to gain reproducible waveforms. The analysis time was $100 \mathrm{msec}$, and the bandwidth was 3-5 kHz. Recording electrodes were placed at Erb's point and the left postcentral $\left(\mathrm{C}^{\prime}\right)$, right postcentral $\left(\mathrm{C} 4^{\prime}\right)$, and midline postcentral $\left(\mathrm{Cz}^{\prime}\right)$ regions of the scalp, in reference to the midline frontal $(\mathrm{Fz})$ scalp, according to the 10-20 International System. For the median nerve, latencies of N10 and N20 waves, interpeak latencies, and peak-to-peak amplitude of the N20 component were measured. For tibial nerve SEPs, latency and peak-to-peak amplitude of the P40 component were measured.

Normal limits were defined (using standardized values determined from a healthy cohort) as an interpeak N10N20 latency of $19.2 \pm 0.9 \mathrm{msec}$, an N20 component amplitude of $1.3 \pm 0.6 \mu \mathrm{V}$, a P40 latency of $38.8 \pm 2.0 \mathrm{msec}$, and a P40 component amplitude of $1.8 \pm 1.3 \mu \mathrm{V} .23$

Evaluation of the SEP measurements was performed by a neurological consultant who was blinded to the clinical course of the patients. Results were dichotomized as present or missing cortical responses and normal or prolonged latencies for each nerve and side. Similar to the determination of a score proposed by Firsching and Frowein ${ }^{8}$ and to better reproduce the integrity of the whole nervous system, each normal result was given 1 point. The points then were grouped and summed to result in a score that ranged from 0 to 8 for each measurement $(4 \times$ cortical responses and $4 \times$ latencies) and used for statistical analysis.

\section{Statistical Analysis}

Statistics were analyzed using IBM SPSS v23 and v24 (IBM Corp.) and R v2.3.5 (R Foundation). For basic analysis of SEP latencies, the Student t-test was used, and univariate ANOVA was used for outcome analysis. Concerning dichotomized and summarized variables, we used multiple binary regression analysis with outcomes dichotomized as good (GOS score of 4 or 5) or poor (GOS score of 1,2, or 3). In the analysis of outcome prediction, we always used the GOS score at the 6-month follow-up visit as the outcome variable. To evaluate the sensitivity and specificity of the predictive variables, we used receiver operating characteristic (ROC) curves. The results were calculated with corresponding $95 \%$ confidence intervals. Significance was defined as a $\mathrm{p}$ value $\leq 0.05$.

\section{Ethics Statement}

In accordance with local and institutional laws and dataprotection regulations, approval by the local ethics committee was obtained and archived. The study was performed in accordance with the ethical standards provided in the 1964 Declaration of Helsinki and its later amendments. 
TABLE 1. Patient characteristics

\begin{tabular}{lc}
\hline \multicolumn{1}{c}{ Characteristic } & Value \\
\hline No. of patients & 48 \\
\hline Age range in yrs (mean \pm SD) & $28-81(58 \pm 11.9)$ \\
\hline Sex & $14(29.2)$ \\
\hline Male & $34(70.8)$ \\
\hline Female & $5-75(31.4 \pm 15.4)$ \\
\hline Length of stay in days, range (mean \pm SD) & \\
\hline Initial presentation & $22(45.8)$ \\
\hline HH grade & $13(27.1)$ \\
\hline III & $13(27.1)$ \\
\hline IV & $9(18.8)$ \\
\hline V & $39(81.3)$ \\
\hline Fisher grade & $12(25.0)$ \\
\hline 3 & $36(75.0)$ \\
\hline 4 & $30(62.5)$ \\
\hline Treatment & $15(31)$ \\
\hline Aneurysm clipping & $12(25)$ \\
\hline Endovascular & \\
\hline Delayed cerebral ischemia & \\
\hline Aneurysm location & \\
\hline Anterior cerebral arteries & \\
\hline Posterior cerebral arteries & \\
\hline Internal carotid arteries & \\
\hline Vas & \\
\hline
\end{tabular}

Values are number (\%) of patients unless otherwise stated.

\section{Results}

A total of 48 patients with high-grade aneurysmal SAH (HH Grade III or higher) were enrolled in the study. This total included 22 patients whose status was HH Grade III, 13 whose status was HH Grade IV, and 13 whose status was $\mathrm{HH}$ Grade V. Their mean age was 58 years (range 28-81 years); 34 patients were female, and 14 were male. The median length of stay was 31.4 days (range 5-75 days). Twelve patients underwent aneurysm clipping, and 36 were treated using endovascular means (Table 1).

At discharge, outcome was deemed good in $27.1 \%$ (13 of 48 ) of the patients and poor in $72.9 \%$ (35 of 48 ); $10.4 \%$ ( 5 of 48 ) of the patients died before discharge. At the follow-up visit, outcomes were good in $60.4 \%$ (29 of 48) of the patients and poor in $39.6 \%(n=19)$. One patient with a GOS score of 2 at discharge died within the follow-up period; compared with their GOS scores at discharge, the scores in all of the other patients had either stabilized or improved (56.3\% [27 of 48]) (Table 2).

The first measurements of SEPs were made in 46 patients ( $2 \pm 1$ day after hemorrhage), and the second measurement was made in 41 patients $(14 \pm 1$ day after hemorrhage). Primary measurements were not obtained in 2 patients and secondary measurements were not obtained in 7 patients because of compliance issues. Thirty-nine $(81.3 \%)$ patients received sedation (propofol and midazolam) at the first SEP measurement, and this number was reduced at the second measurement to 6 (12.5\%). We found no significant differences in absolute SEP latencies or cor-
TABLE 2. GOS scores at discharge and 6-month follow-up visit

\begin{tabular}{crrrrr}
\hline \multirow{2}{*}{$\begin{array}{c}\text { GOS Score } \\
\text { at Discharge }\end{array}$} & \multicolumn{5}{c}{ 6-Mo Postop GOS Score (\% [no.] of patients) } \\
\cline { 2 - 6 } & 1 & 2 & 3 & 4 & 5 \\
\hline 1 & $100.0(5)$ & & & & \\
\hline 2 & $14.3(1)$ & $42.9(3)$ & $42.9(3)$ & & \\
\hline 3 & & & $30.4(7)$ & $47.8(11)$ & $21.7(5)$ \\
\hline 4 & & & & $20.0(2)$ & $80.0(8)$ \\
\hline 5 & & & & & $100.0(3)$ \\
\hline
\end{tabular}

tical responses between patients with and those without sedation in a 1-way ANOVA for Measurements 1 and 2. Also, the results of multivariate (see below) and univariate regressions (Measurement $1, \mathrm{~B}=-1.5, \mathrm{p}=0.07$; Measurement $2, \mathrm{~B}=-0.2, \mathrm{p}=0.84$ ) did not confirm a significant impact of sedation on calculated SEP scores.

In the first SEP measurements, the mean N20 latencies were $20.87 \pm 1.53 \mathrm{msec}$ (range $18.60-25.30 \mathrm{msec}$ ) on the left side and $20.74 \pm 1.41 \mathrm{msec}$ (range 18.40-25.20 msec) on the right side. The mean N10-N20 latencies were 10.19 $\pm 1.42 \mathrm{msec}$ (range $8.70-13.90 \mathrm{msec}$ ) on the left side and $10.07 \pm 1.05 \mathrm{msec}$ (range $8.20-13.40 \mathrm{msec}$ ) on the right side. The mean P40 latencies were $44.36 \pm 5.12 \mathrm{msec}$ (range 35.40-58.70 msec) on the left side and $43.96 \pm 3.68$ msec (range $39.60-56.70 \mathrm{msec}$ ) on the right side.

In the second SEP measurements, the mean N20 latencies were $20.2 \pm 1.35 \mathrm{msec}$ (range 17.90-23.00 $\mathrm{msec}$ ) on the left side and $23.40 \pm 1.28 \mathrm{msec}$ (range 18.10-23.40 $\mathrm{msec}$ ) on the right side. The mean N10-N20 latencies were $9.73 \pm 1.13 \mathrm{msec}$ (range $7.70-12.90 \mathrm{msec}$ ) on the left side and $9.79 \pm 1.11 \mathrm{msec}$ (range $7.30-213.00 \mathrm{msec}$ ) on the right side. The mean $\mathrm{P} 40$ latencies were $43.73 \pm 4.96 \mathrm{msec}$ (range 37.70-57.80 $\mathrm{msec}$ ) on the left side and $43.48 \pm 3.90$ msec (range $37.70-56.10 \mathrm{msec}$ ) on the right side.

In Measurement 1, we found mean N20 SEP amplitudes of $2.49 \pm 2.06 \mu \mathrm{V}$ (range $9.20-0.20 \mu \mathrm{V}$ ) on the left side and $3.02 \pm 2.06 \mu \mathrm{V}$ (range 9.70-0.20 $\mu \mathrm{V}$ ) on the right side. In Measurement 2, the mean N20 SEP amplitudes were 3.41 $\pm 2.84 \mu \mathrm{V}$ (range $12.90-0.20 \mu \mathrm{V}$ ) on the left side and 4.69 $\pm 3.63 \mu \mathrm{V}$ (range $20.60-0.50 \mu \mathrm{V}$ ) on the right side.

The mean P40 SEP amplitudes in Measurement 1 were $1.90 \pm 1.55 \mu \mathrm{V}$ (range $7.40-0.30 \mu \mathrm{V})$ on the left side and $1.80 \pm 1.33 \mu \mathrm{V}$ (range $5.50-0.40 \mu \mathrm{V}$ ) on the right side. In Measurement 2, the mean P40 SEP amplitudes were 4.05 $\pm 6.65 \mu \mathrm{V}$ (range $25.40-0.30 \mu \mathrm{V}$ ) on the left side and 3.41 $\pm 5.36 \mu \mathrm{V}$ (range $23.90-0.10 \mu \mathrm{V}$ ) on the right side.

\section{Analysis}

When only the presence of cortical responses was analyzed, no patient with a GOS score of 4 or 5 at follow-up had missing responses on median SEPs in Measurement 1 or 2 , whereas in the group of patients with a GOS score of 1,2 , or $3,35.7 \%$ and $28.6 \%$ had missing responses on median SEPs in Measurements 1 and 2, respectively ( $\mathrm{p}$ $=0.014)$. Cortical responses for the lower extremities did not correlate with clinical outcome in Measurement 1, but in Measurement 2, 7.7\% in the good-outcome group had at least 1 missing response versus $64.3 \%$ in the poor-outcome group $(\mathrm{p}=0.001)$. 


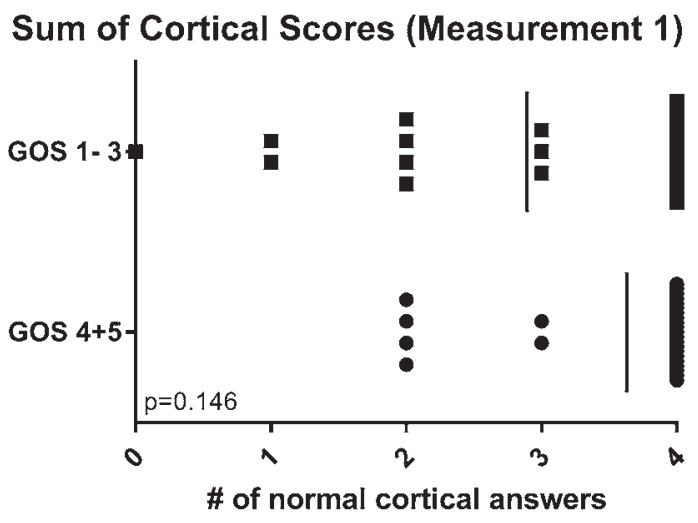

Sum of Cortical Scores (Measurement 2)

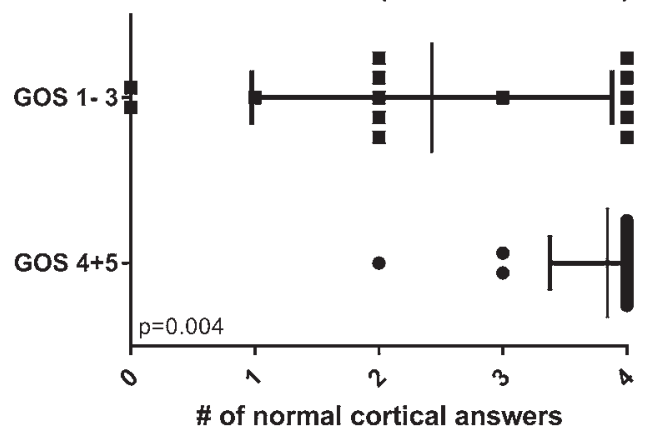

Sum of Latency Scores (Measurement 1)

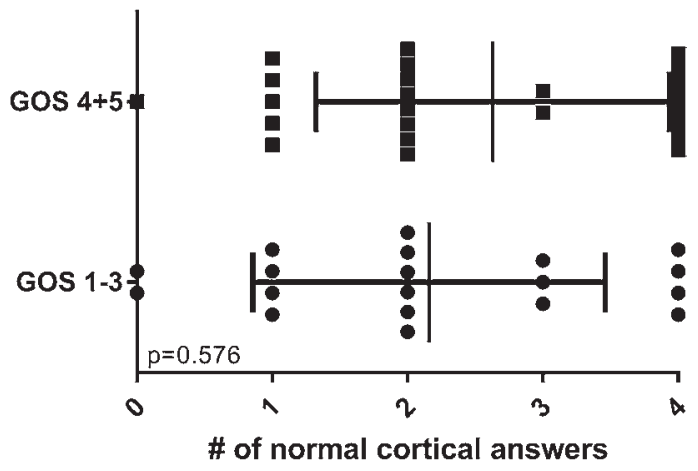

Sum of Latency Scores (Measurement 2)

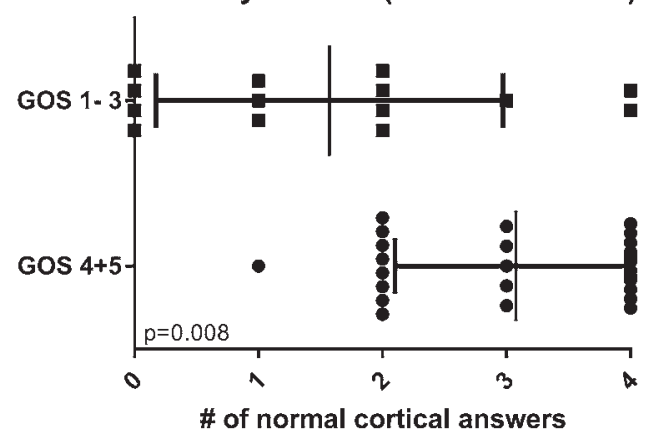

FIG. 1. Calculated scores for cortical answers and latencies in the first and second SEP measurements according to GOS score.

When we analyzed the latencies of the arms at Measurement 1 , we identified no significant differences between outcome groups, whereas in Measurement 2, no patient in the good-outcome group had abnormal latencies in both arms, and $18.5 \%$ had abnormal latencies in $1 \mathrm{arm}$. In contrast, $64.3 \%$ in the poor-outcome group had abnormal latencies, $28.6 \%$ in both arms and $35.7 \%$ in $1 \mathrm{arm}(\mathrm{p}=$ $0.003)$. In the lower extremities, $23 \%$ in the good-outcome group had abnormal latencies in both legs and $19.2 \%$ had abnormal latencies in $1 \mathrm{leg}$, whereas in the poor-outcome group, $64.3 \%$ had abnormal latencies in both legs and $21.4 \%$ had them in 1 leg $(\mathrm{p}=0.034)$.

One-way ANOVA did not identify any significant correlation between P40 or N20 amplitudes or latencies and clinical outcome, regardless of the measurement point, and the intergroup differences were not significant.

\section{Calculated SEP Scores}

Patients with a GOS score of 1 or 2 had median scores of 4 (range 2-7) in the first measurement and 1 (range 0-2) in the second measurement. Patients with a GOS score of 3 had median scores of 5 (range 2-7) in the first measurement and 6 (range 0-7) in the second measurement. Patients with a GOS score of 4 or 5 had median scores of 6 (range 3-7) in the first measurement and 7 (range 4-8) in the second measurement.

In summary, in regard to cortical and latency scores, we found no significant difference in the score distributions when patient outcomes were dichotomized in cross tables and tested by Pearson's chi-square test for Measurement 1 (Fig. 1). However, in Measurement 2, a significant difference in the distributions of both scores was identified (cortical responses, $\mathrm{p}=0.004$; latency, $\mathrm{p}=0.008$; chi-square) (Fig. 1). The mean scores in both cases were significantly higher in the good-outcome group than in the poor-outcome group (latencies in Measurement 2, 3.1 vs 1.6 points, respectively [p $=0.002]$; cortical responses in Measurement 2, 3.8 vs 2.4, respectively [p $<0.001$, t-test]).

When we analyzed cumulative cortical and latency scores, we identified no significant correlation between values in Measurement 1 and patient outcome. In multiple binary backward regression including the variables cumulative score, patient age, HH grade, GCS score, and presence of sedation, the only variable associated with poor outcome was the cumulative scores for Measurement 2 (OR 0.39, p = 0.005) (Fig. 2), the impact of which was confirmed in forward binary regression.

For the identification of parameters associated with patient outcome dichotomized as poor or good, we performed an ROC analysis in which $\mathrm{HH}$ grades had an area under the curve (AUC) of 0.71 (95\% CI 0.56-0.85, p = 0.02 ), and GCS scores on admission had an AUC of 0.66 (95\% CI 0.50-0.82, $\mathrm{p}=0.07$ ). Median SEPs in the first measurement had an AUC of 0.65 (95\% CI 0.49-0.85, p $=0.10)$ and in the second measurement an AUC of 0.76 (95\% CI 0.61-0.91, $\mathrm{p}<0.01$ ). Tibial nerve SEPs in the first measurement had an AUC of 0.61 (95\% CI 0.49-0.80, p $=0.22)$ and in the second measurement an AUC of 0.81 (95\% CI 0.66-0.95, p < 0.01) (Fig. 3).

Overall SEPs in the first measurement had an AUC of $0.64(95 \%$ CI $0.47-0.80, \mathrm{p}=0.11)$ and in the second measurement an AUC of 0.82 (95\% CI 0.68-0.97, p < 0.01). 


\section{Cumulative Scoring Measurement 1}

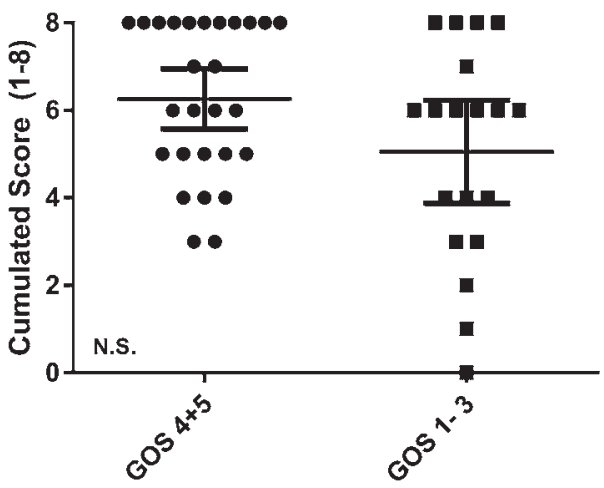

Outcome

\section{Cumulative Scoring Measurement 2}

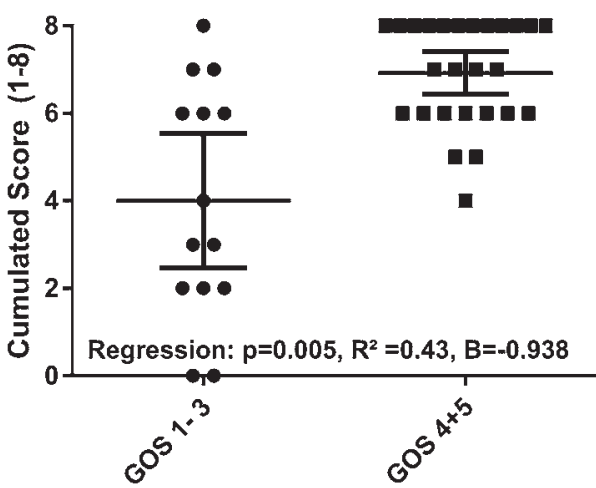

Outcome

FIG. 2. Cumulative scores in the first and second SEP measurements according to GOS score. N.S. = not significant.

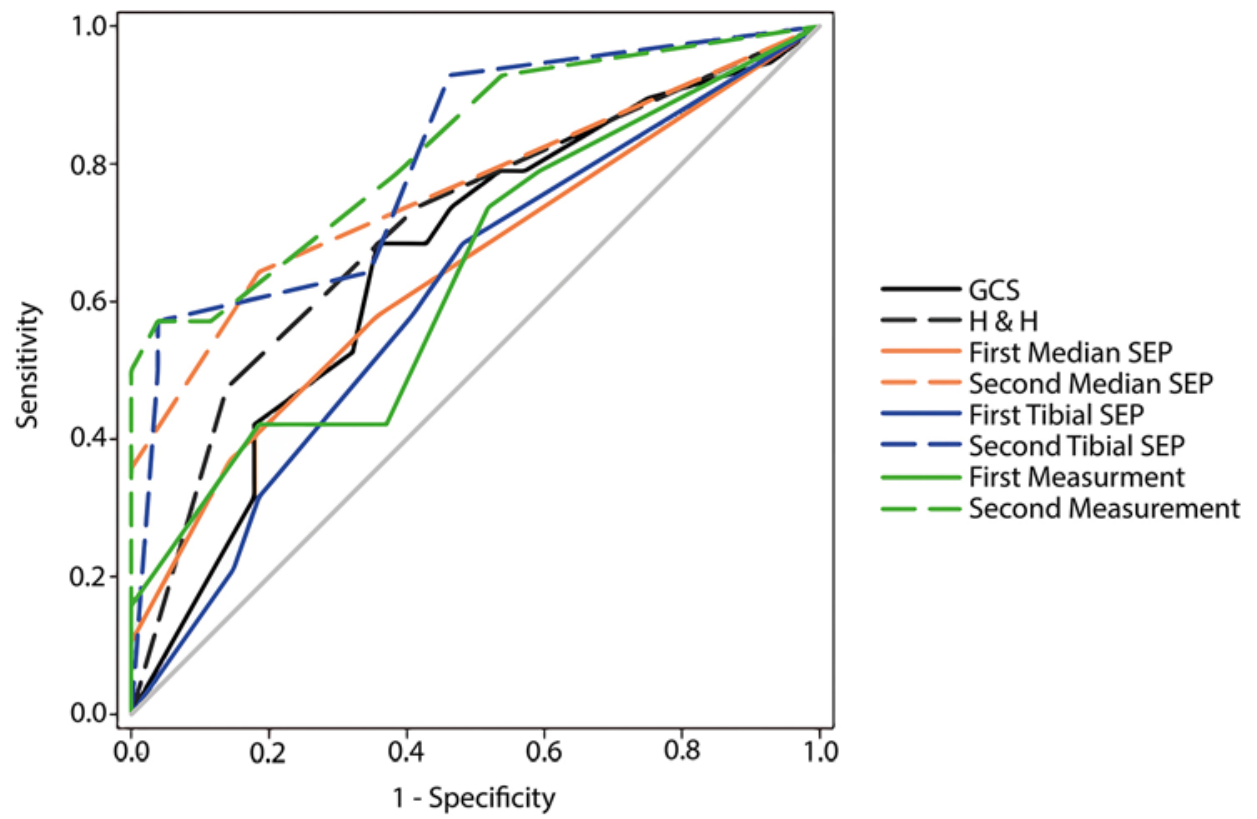

\begin{tabular}{lcccc} 
Test Variables & GCS & H \& H & 1st Measurement 2nd Measurement \\
\hline ROC Curve Area & 0.66 & 0.71 & 0.64 & 0.82 \\
Standard Error & 0.08 & 0.07 & 0.08 & 0.07 \\
95\% Confidence Interval & $0.50-0.82$ & $0.56-0.85$ & $0.47-0.80$ & $0.68-0.97$ \\
P Value & 0.07 & 0.02 & 0.11 & $<0.01$ \\
Sample Size - 0 & 28 & 29 & 27 & 26 \\
Sample Size - 1 & 19 & 19 & 19 & 14 \\
& & & & \\
Test Variables & 1st Median SEP & 2nd Median SEP & 1st Tibial SEP & 2nd Tibial SEP \\
\hline ROC Curve Area & 0.64 & 0.76 & 0.61 & 0.81 \\
Standard Error & 0.08 & 0.08 & 0.08 & 0.07 \\
95\% Confidence Interval & $0.49-0.80$ & $0.61-0.91$ & $0.45-0.77$ & $0.66-0.95$ \\
P Value & 0.10 & 0.01 & 0.22 & $<0.01$ \\
Sample Size - 0 & 28 & 27 & 27 & 26 \\
Sample Size - 1 & 19 & 14 & 19 & 14
\end{tabular}

FIG. 3. ROC curve assessing predictive values of the first and second SEP measurements along with the GCS score and $\mathrm{HH}$ $(\mathrm{H} \& \mathrm{H})$ grade for a poor outcome. The predictive value is represented by the AUC. 


\section{Vasospasm}

Vasospasm, as detected by transcranial Doppler ultrasonography, CT, or conventional angiography, was apparent at least once during the treatment of $68.8 \%$ (33 of 48) of the patients. Patients with poor outcome experienced vasospasm significantly more often than those with good outcome (84.2\% vs $58.6 \%$, respectively; OR 3.8; $\mathrm{p}=0.05)$. If delayed cerebral ischemia resulted from vasospasm (overall rate $62.5 \%$ ), this effect was even more obvious (89.5\% vs 44.8\%, respectively; OR 10.5; $\mathrm{p}<0.01)$.

\section{Discussion}

SEPs as an electrophysiological diagnostic tool have been well described in the context of outcome prediction in comatose patients after cardiac arrest or traumatic brain injury. 5,15,18,20 The main use of SEPs in these series was for the prediction of the likelihood of not awakening from coma, and the most important predictor seems to be the absence of cortical responses (N20 waves). We analyzed SEPs in a cohort of patients with high-grade $\mathrm{SAH}$, a disease in which patient recovery depends not only on the degree of primary brain damage but also the secondary complications that result from the hemorrhage. In our series, patients who did not recover from coma (GOS score of 1 or 2) had either no cortical response or no more than 1 cortical response with or without normal latency. One patient did not show any cortical responses (SEP score of 0 ) in the second measurement and had a GOS score of 3, which does not support the $100 \%$ specificity found in other series for missing cortical responses always being associated with not awaking from coma. ${ }^{14,15,20}$

Although admitted with a high-grade SAH, most patients in our series had a favorable outcome (GOS score of 4 or 5) at the 6-month follow-up visit. In the second measurement, the presence of bilateral cortical responses was associated with a favorable outcome on median and tibial nerve SEPs (sensitivities $100 \%$ and $92 \%$ and specificities $71 \%$ and $64 \%$, respectively). Bilateral normal latencies also were associated with a favorable outcome on median and tibial nerve SEPs (sensitivities $100 \%$ and $77 \%$, respectively, and specificity $64 \%$ for both). In our multivariate analysis, every cortical response or normal latency was represented with 1 point, following a scoring system for SEPs used by Firsching and Frowein, ${ }^{8}$ which resulted in a score that ranges from 0 to 8 . Our analysis revealed that the score was associated with clinical outcome, and this association was found also when cortical responses or latencies from all extremities and cortical responses and latencies from median or tibial nerves were tested separately (Figs. 2 and 3). We did not involve the amplitude measurement in the calculated score because it is known to be subject to wide interindividual variability ${ }^{19}$ and to avoid further complication of the scoring system.

In some studies, measurement of amplitudes was proposed to be helpful because amplitude reduction in early SEPs was associated with no recovery, whereas latencies did not add prognostic information. ${ }^{13}$ In other studies, prolonged latencies were associated with poor recovery. ${ }^{14,20}$ Neither absolute values of amplitudes nor those of latencies in the first or second measurement correlated with the clinical outcome in our patient cohort, which might underline the difference between SAH and other etiologies of brain damage and supports the finding of Logi et al., ${ }^{13}$ who evaluated SEPs in patients in a coma of different etiologies and concluded that these different etiologies were associated with different electrophysiological patterns of SEPs.

Another goal of this study was to determine the most useful point at which to measure SEPs to gain reliable information about potential recovery in patients with highgrade $\mathrm{SAH}$. We chose the first time point to be within 3 days after hemorrhage, because the primary brain damage that results from SAH should have taken place by then. Each patient in our cohort suffered from a high-grade $\mathrm{SAH}$, and therefore, most of them $(81.3 \%)$ were under sedation during the first SEP measurement. In the study design, to avoid the risk of elevated intracranial pressure, we decided not to interrupt sedation to perform the measurement. When we evaluated median nerve SEPs, the only significant correlation was found in the univariate analysis between the presence of cortical responses and a favorable long-term outcome, because none of the patients with a GOS score of 4 or 5 at the 6-month follow-up had missing cortical responses. This correlation could not be confirmed in the multivariate analysis. Neither the presence of cortical responses nor their latencies on tibial nerve SEPs were informative regarding outcome.

SEPs are known to be relatively robust to the influence of anesthetic agents, ${ }^{3}$ and they are widely used during intraoperative monitoring.,6,22 However, sedatives were found to cause significant reduction of amplitude and prolongation of latencies in SEPs compared with SEPs measured in awake patients,, 912 which, from our point of view, might limit the use of SEPs for outcome prediction in patients under sedation. However, this fact does not explain why early SEP measurement was not conclusive in our study, because the presence of cortical responses, which should not be affected by sedation, was not associated with clinical outcome in the multivariate analysis. Moreover, univariate and multivariate regressions did not reveal an impact of sedation on calculated SEP scores. A more convincing explanation would be the fact that delayed cerebral ischemia resulting from vasospasm took place in $62.5 \%$ of the patients within the first 2 weeks and affected their outcome significantly (OR 10.5, p < 0.01). Therefore, we recommend SEP measurements to be made 2 weeks after hemorrhage to gain reliable information about long-term outcome.

Because of the intensive care unit setting and the difficulties in positioning patients at risk for developing elevated intracranial pressure, recording of N22 SEPs at the lumbar level was not performed, which probably led us to miss prolonged latencies of the P40 component that resulted from peripheral nerve damage, which limits the interpretation of latencies in the measurement of tibial nerve SEPs in this series; however, none of the included patients suffered from trauma or a previously known myelopathy or neuropathy. Nevertheless, measurement of tibial nerve SEPs was valuable in this series, because not only normal or prolonged latencies but also the presence or absence of cortical responses were associated with patient outcome. In addition, the calculated score on the second measurement 
of tibial nerve SEPs had a high sensitivity and specificity for outcome prediction (AUC 0.81) and thus performed better than median nerve SEPs (AUC 0.76). The interpretation of tibial nerve SEPs toward the ability of patients to move independently would be interesting but was not addressed in this series because of the limited value of the applied outcome measurement in this regard.

In addition to the relatively small number of the included patients in this study, the missing first SEP measurement in 2 patients and the second measurement in 7 patients are limitations that must be considered when interpreting our results.

\section{Conclusions}

Early SEP measurement after SAH was not predictive of outcome in our patient cohort, but SEP measurements of the median and tibial nerves 2 weeks after hemorrhage were associated with long-term outcome in patients with high-grade SAH and might be useful in clinical practice for estimating recovery potential in this group of patients.

\section{References}

1. Agarwal N, Hamilton DK, Ozpinar A, Choi P, Hart R, Yaylali I: Intraoperative neurophysiological monitoring for adult patients undergoing posterior spinal fusion. World Neurosurg 99:267-274, 2017

2. Al-Rawi MA, Hamdan FB, Abdul-Muttalib AK: Somatosensory evoked potentials as a predictor for functional recovery of the upper limb in patients with stroke. J Stroke Cerebrovasc Dis 18:262-268, 2009

3. Amantini A, Fossi S, Grippo A, Innocenti P, Amadori A, Bucciardini L, et al: Continuous EEG-SEP monitoring in severe brain injury. Neurophysiol Clin 39:85-93, 2009

4. Bederson JB, Connolly ES Jr, Batjer HH, Dacey RG, Dion JE, Diringer MN, et al: Guidelines for the management of aneurysmal subarachnoid hemorrhage: a statement for healthcare professionals from a special writing group of the Stroke Council, American Heart Association. Stroke 40:994-1025, 2009

5. Claassen J, Hansen HC: Early recovery after closed traumatic head injury: somatosensory evoked potentials and clinical findings. Crit Care Med 29:494-502, 2001

6. Eager M, Shimer A, Jahangiri FR, Shen F, Arlet V: Intraoperative neurophysiological monitoring (IONM): lessons learned from 32 case events in 2069 spine cases. Am J Electroneurodiagn Technol 51:247-263, 2011

7. Feys H, Van Hees J, Bruyninckx F, Mercelis R, De Weerdt $\mathrm{W}$ : Value of somatosensory and motor evoked potentials in predicting arm recovery after a stroke. J Neurol Neurosurg Psychiatry 68:323-331, 2000

8. Firsching R, Frowein RA: Multimodality evoked potentials and early prognosis in comatose patients. Neurosurg Rev 13:141-146, 1990

9. Grundy BL: Intraoperative monitoring of sensory-evoked potentials. Anesthesiology 58:72-87, 1983

10. Hunt WE, Hess RM: Surgical risk as related to time of intervention in the repair of intracranial aneurysms. J Neurosurg 28:14-20, 1968

11. Jennett B, Bond M: Assessment of outcome after severe brain damage. Lancet 1:480-484, 1975

12. Kochs E, Wüst P, Blanc I, Schulte am Esch J: [Acoustic and somatosensory evoked cortical potentials in sedation with midazolam and drug antagonism with flumazenil.] Anasth Intensivther Notfallmed 24:49-56, 1989 (Ger)
13. Logi F, Fischer C, Murri L, Mauguière F: The prognostic value of evoked responses from primary somatosensory and auditory cortex in comatose patients. Clin Neurophysiol 114:1615-1627, 2003

14. Madl C, Kramer L, Domanovits H, Woolard RH, Gervais H, Gendo A, et al: Improved outcome prediction in unconscious cardiac arrest survivors with sensory evoked potentials compared with clinical assessment. Crit Care Med 28:721-726, 2000

15. Madl C, Kramer L, Yeganehfar W, Eisenhuber E, Kranz A, Ratheiser K, et al: Detection of nontraumatic comatose patients with no benefit of intensive care treatment by recording of sensory evoked potentials. Arch Neurol 53:512-516, 1996

16. Prohl J, Röther J, Kluge S, de Heer G, Liepert J, Bodenburg S, et al: Prediction of short-term and long-term outcomes after cardiac arrest: a prospective multivariate approach combining biochemical, clinical, electrophysiological, and neuropsychological investigations. Crit Care Med 35:1230-1237, 2007

17. Rinkel GJ, Algra A: Long-term outcomes of patients with aneurysmal subarachnoid haemorrhage. Lancet Neurol 10:349-356, 2011

18. Robinson LR, Micklesen PJ, Tirschwell DL, Lew HL: Predictive value of somatosensory evoked potentials for awakening from coma. Crit Care Med 31:960-967, 2003

19. Scarpino M, Lanzo G, Carrai R, Lolli F, Migliaccio ML, Spalletti M, et al: Predictive patterns of sensory evoked potentials in comatose brain injured patients evolving to brain death. Neurophysiol Clin 47:19-29, 2017

20. Sherman AL, Tirschwell DL, Micklesen PJ, Longstreth WT Jr, Robinson LR: Somatosensory potentials, CSF creatine kinase BB activity, and awakening after cardiac arrest. Neurology 54:889-894, 2000

21. Teasdale G, Jennett B: Assessment of coma and impaired consciousness. A practical scale. Lancet 2:81-84, 1974

22. Tsai SW, Tsai CL, Wu PT, Wu CY, Liu CL, Jou IM: Intraoperative use of somatosensory-evoked potential in monitoring nerve roots. J Clin Neurophysiol 29:110-117, 2012

23. Waberski TD: Somatosensorisch evozierte Potenziale (SEP), in Buchner H, Noth J (eds): Evozierte Potenziale, Neurovegetative Diagnostik, Okulographie. Stuttgart: Thieme, 2005, pp 21-28

24. Zhang Y, Su YY, Ye H, Xiao SY, Chen WB, Zhao JW: Predicting comatose patients with acute stroke outcome using middle-latency somatosensory evoked potentials. Clin Neurophysiol 122:1645-1649, 2011

\section{Disclosures}

The authors report no conflict of interest concerning the materials or methods used in this study or the findings specified in this paper.

\section{Author Contributions}

Conception and design: Abboud. Acquisition of data: Gelderblom, Schwarz, Czorlich. Analysis and interpretation of data: Abboud, Mende. Drafting the article: Abboud, Mende. Critically revising the article: Abboud, Schmidt, Regelsberger, Westphal. Reviewed submitted version of manuscript: all authors. Approved the final version of the manuscript on behalf of all authors: Abboud. Statistical analysis: Mende, Vettorazzi. Study supervision: Abboud.

\section{Correspondence}

Tammam Abboud, Department of Neurosurgery, University Medical Center Göttingen, Robert-Koch-Strasse 40, Göttingen 37075, Germany. email: tammamabboud@gmail.com. 